\title{
Balance y propuestas para una Administración Pública al servicio de la Comunidad Organizada
}

Comisión Estado y Administración Pública del Instituto Patria

... a una concepción que puede ser más o menos buena se la completa y se la realiza con una ejecución inteligente (...) Nadie puede gobernar lo inorgánico. Es necesario, antes de gobernar, de dirigir o de mandar, tener algo orgánico para hacer (...) La organización que me interesa es el hombre caminando, comiendo y haciendo su trabajo. Lo mismo pasa con la organización institucional. No es bastante ese lindo cuadro. No. Es mejor que no sea tan lindo y que ande, que ande en la realidad, con sus enfermedades, con sus pasiones y con todos los defectos $y$ virtudes que los hombres llevan a la organización (...) ¿A dónde va un hombre que no tenga sentimientos ni pensamiento? ¿Y adónde iría una Nación que no tuviese un pensamiento y un sentimiento comunes?

El gobierno no puede ser la acción burocrática del trámite: el gobierno tiene que ser algo más noble (...) Muchas veces el Gobierno crea un callo sobre el corazón. Eso es lo que debemos evitar: que no haya callos ni sobre el entendimiento. Nosotros tenemos una responsabilidad enorme que cumplir. Estamos de acuerdo en enfrentarla y afrontarla. De cómo lo hagamos, es de lo que nos va a pedir cuentas el futuro de la Nación.

Juan. D. Perón ${ }^{1}$ 


\section{Introducción}

Este documento es fruto de la reflexión y el trabajo de la Comisión de Estado y Administración Pública del Instituto Patria durante el complejo 2020, y busca avanzar en la elaboración de conceptos que den forma a una gestión pública de dimensión comunitaria, cultural y formativa, colaborando con la construcción de capacidades estatales situadas. ${ }^{2}$ Anhelamos un Estado de lo común, de los proyectos colectivos, de la solidaridad, del cuidado y de los acuerdos. Una concepción ajena a los paradigmas individualistas, para transformar la forma de concebir lo público.

Esta concepción pone en el centro de la escena a las organizaciones sociales y a las y los trabajadores estatales, para convertir al Estado en el ámbito donde tramitar las esperanzas y los intereses del Pueblo, donde concebir y hacer realidad un proyecto de Nación soberana. Un Estado que sea memoria e identidad, garante de derechos y creador de oportunidades. Un Estado capaz de conducir el desarrollo con inclusión e integración a partir de nuestras banderas históricas:justicia social, soberanía política e independencia económica. Un Estado que sea, efectivamente, público. Que logre mayor alcance y eficacia en sus estrategias y políticas. Que tenga la capacidad para fijarse metas a la altura de los anhelos humanos.

El entramado estatal se despliega en un marco federal donde la Nación, las provincias y los municipios deben confluir para que el desarrollo sea territorialmente equitativo, superando asimetrías regionales centenarias. El federalismo requiere la articulación virtuosa de las construcciones político-territoriales. Nuestras administraciones estatales deben protagonizar la multipolaridad regional y el desarrollo local reduciendo brechas económicas, sociales, políticas y culturales.

Hemos decidido abordar la planificación e implementación de políticas, el acceso y la simplificación de los procesos administrativos, propuestas de evaluación integrales y situadas, la formación de dirigentes y la promoción de trabajadores/as para que protagonicen el heroísmo de lo cotidiano. Estos son los imperativos para transformar una realidad desigual e injusta. En este marco, las siguientes propuestas y recomendaciones son el punto de llegada de nuestros debates, para el inicio de un diálogo que las enriquezca y las haga efectivas.

\section{En torno a las capacidades estatales}

\section{Capacidades político estratégicas y modelos de desarrollo}

Creemos en la necesidad de orientar la organización estatal, como lo prevé el Presupuesto para la Administración Nacional 2021, hacia un modelo de desarrollo sustentable en lo económico, social y político, a partir de cinco condiciones estructurales: integración, dinamismo, estabilidad, federalismo y soberanía. Para alcanzarlo, debemos redoblar los esfuerzos 
de coordinación entre los niveles estatales mediante una planificación situada que tenga presente los acuerdos políticos y priorice el manejo de conflictos como una función específica.

Esta planificación requiere de diseños institucionales y políticas que mejoren la vinculación con los sujetos productivos y sociales con menor capacidad de lobby, quienes requieren de asistencia técnica y financiera para desarrollarse en una coyuntura marcada por la destrucción productiva del gobierno saliente y agravada por la pandemia. Desplegar las capacidades estatales en este escenario requiere incorporar, en mesas y ámbitos permanentes, las demandas de los actores sociales promotores del desarrollo, acercando el Estado mediante una gestión transparente y de acceso directo.

Una planificación de gobierno, política y situada, supone: 1) desarrollar capacidades de planeamiento estratégico situacional (definición de horizontes utópicos, lectura de la realidad, cálculo de viabilidad, salas de situación, acuerdos estratégicos); 2) federalizar la intervención estatal para potenciar el desarrollo territorial; 3) concertar entre instituciones públicas y privadas, con anclaje en cada territorio; 4) promover los emprendimientos sociales, culturales y productivos; 6) tramitar con agilidad los proyectos del desarrollo, transparentando y facilitando el acceso a los instrumentos de promoción.

Para este cometido, las capacidades político estratégicas, de gestión, relacionales y económicas que deben fortalecerse son muy diversas. Entre ellas, sobresalen las capacidades para expresar proyectos de desarrollo territorial (regional, nacional, provincial, local) y temáticos (ambiental, educativo, derechos de las mujeres, infancias, otros), las que se complementan con capacidades de liderazgo para la conducción y el gobierno. Creemos que los proyectos estratégicos (territoriales y temáticos), así como la voluntad política que los orienta, son motor de la gestión pública. A estas capacidades centrales se suman las referidas a la información y la comunicación pública. Es necesario ampliar el criterio de "información pública" hacia el de "información de interés público" o "de interés social", que no solo incluye la información sobre acciones estatales, sino también sobre aquellas desarrolladas por otros entes que afectan directamente a la sociedad al punto de poner en riesgo a la población o afectar sus intereses.

\section{Capacidades para una gestión federal}

Valoramos las acciones que se están desarrollando para mejorar la coordinación entre Nación-Provincias-Municipios, muchas de las cuales se insertaron en la agenda político-estatal a partir del trabajo del Instituto Patria, entre ellas:

- Ley de capitales alternas, una herramienta estratégica para la puesta en valor del interior y la construcción de potentes instrumentos de lógica federal, 
tales como: a) los Gabinetes Federales, consistentes en la reunión de autoridades nacionales y de cada provincia para planificar acciones conjuntas, que deben complementarse con las "Conferencias Federales" que promuevan un trabajo sistemático de las autoridades del Pacto Federal; y b) la relocalización de oficinas del Estado Nacional, de forma tal que se acerquen a los actores sociales y políticos involucrados en su tarea sustantiva.

\section{- Acceso de las provincias y municipios a las} tecnologías, a través acciones que el gobierno nacional está desplegando en el marco de políticas que promueven la soberanía tecnológica como herramienta de trabajo y comunicación.

Hacia el futuro, se requiere avanzar en la construcción y el fortalecimiento de las capacidades estatales federales, potenciar las funciones del Consejo Federal de Inversiones (CFI), consolidar y jerarquizar los Consejos Federales, promover Acuerdos Regionales Metropolitanos, atender a la cuestión de la tierra y los grandes conglomerados poblacionales y fortalecer la formación federal, a través de una Ley Nacional de Formación Pública (ver párrafo final: Ley Scalabrini Ortiz).

\section{Capacidades institucionales y regulatorias}

Los marcos regulatorios y procedimentales condicionan el ritmo y los límites de la acción estatal, pudiendo ser facilitadores del acceso y la tutela administrativa efectiva o, por el contrario, convertirse en un factor más de exclusión. Las capacidades institucionales y sus regulaciones, su identificación, mejora, comunicación y capacitación de sus operadores, deben atenderse transversalmente, mediante una gestión colaborativa basada en la interacción entre las distintas jurisdicciones y la sociedad en su conjunto.

$\mathrm{Al}$ interior de las organizaciones estatales, es necesario mejorar los procesos mediante una reingeniería que elimine actuaciones administrativas que no agregan valor, determine con claridad las unidades de responsabilidad primaria, y acote las intervenciones administrativas a las que resulten indispensables para obtener decisiones estatales legítimas, válidas, racionales, proporcionales y socialmente valoradas. Las nuevas tecnologías y sistemas de información y gestión permiten repensar los procesos abandonando paulatinamente el modelo de gestión secuencial, propio del soporte papel, para adoptar mecanismos simultáneos y colaborativos de gestión digital en línea. Este camino debe recorrerse desde la soberanía tecnológica, sin tercerizar los procesos administrativos, moneda corriente del gobierno anterior que 
colonizó el mapa estatal con empresas que vendieron software propietario y servicios anexos, privatizando lo público y generando negocios sin controles ni regulaciones.

En la interacción con la sociedad, es indispensable realizar el principio de colaboración, previendo espacios de atención únicos, presenciales y virtuales, que simplifiquen y mejoren la experiencia de quienes acuden ante la administración pública, avanzando en la igualdad efectiva de resultados a través de un asesoramiento y procuración estatal destinado a quienes no poseen los medios para costear un patrocinio letrado o, en el caso de organizaciones comunitarias o PyMEs, no dispongan de las estructuras administrativas adecuadas para gestionar procesos complejos.

\section{Capacidades administrativas: procedimientos de compras y contrataciones}

La mejora y simplificación de los procesos de compras y contrataciones estatales, acortando los tiempos de gestión sin afectar la concurrencia y la transparencia, es imprescindible para sostener el principio de la licitación pública que se ve amenazado por la lógica de la emergencia administrativa permanente y la contratación directa. Las múltiples exigencias formales extienden los procesos y dificultan la concurrencia de proveedores que carecen de la estructura administrativa necesaria, especialmente las PyMEs, las universidades y los emprendedores sociales. Es necesario revisar los procesos fraguados al calor del financiamiento de programas internacionales que, bajo el manto de la competencia y la transparencia, tienden a favorecer a las grandes empresas transnacionales a través de la privatización de los programas que se utilizan. En nombre de la despapelización, se ha privatizado la tramitación pública, un tema que deberemos abordar y revertir

Es posible agilizar procesos licitatorios y hacerlos más efectivos, adaptándolos a las características de cada organización, entendiendo que la compra centralizada puede ser adecuada en ciertos casos, mientras que, en otros, lo será la gestión descentralizada. Además, ciertas organizaciones públicas operan en mercados muy específicos de productos no genéricos, que obligan a trabajar con gran rigurosidad técnica sus especificaciones contractuales, por lo que deben tener un plan de adquisiciones acorde a sus necesidades y una política de desarrollo de proveedores.

Es necesario, además, aprovechar el poder de compra estatal para motorizar al sistema productivo, fortaleciendo los acuerdos entre organismos que fomentan la investigación tecnológica y aquellos que requieren determinados productos.

En conclusión, los procesos de compra tienen especificidades según el sector de política pública donde interviene cada organismo -sus tiempos, las necesidades y las características de cada servicio, y esto obliga a abandonar el enfoque de especialización normativa de las áreas de compras para integrarlas al plan de gestión de la organización. 
El compre argentino en organismos como el PAMI, ANSES, MDS y otras carteras, es, además, un tema central vinculado a las posibilidades del desarrollo económico.

V. Gestión del empleo público

No puede haber un funcionario de ninguna categoría ni un empleado destinado al servicio de la Nación que no piense estrechamente dentro de la doctrina nacional, porque él es el ejecutor directo de esa doctrina...un funcionario o un empleado público, que es el ejecutor director por mandato implícito de la Constitución y de la ciudadanía, no puede estar fuera de eso. ${ }^{3}$

El proceso abierto en 2003 no alcanzó a revertir las consecuencias de las reformas que impactaron en la organización y el perfil del empleo público. A ello, se suma el deterioro producido durante la presidencia de Mauricio Macri, y las consecuencias del aislamiento y trabajo remoto por la pandemia del COVID-19, volviéndonos a encontrar, con los matices de la época, ante un panorama similar al de principios de siglo.

Tenemos la convicción de que los procesos y productos de las organizaciones son esencialmente colectivos, por lo cual es necesario pensar en las trayectorias de las y los trabajadores estatales integrándolas con los proyectos institucionales, y con esa perspectiva encarar los procesos de selección, formación y evaluación. Debemos incorporar la variable del compromiso con los valores de un proyecto de Nación con vocación democrática en las trayectorias de quienes se desempeñan en la administración pública, tanto en su selección, promoción y evaluación, como en el ejercicio de funciones o roles de dirección. En todos los casos, es necesario aplicar mecanismos y acciones afirmativas que garanticen la igualdad de género, y erradiquen cualquier sesgo contra los grupos más postergados.

Por otra parte, se requiere un proceso de regularización de trabajadoras y trabajadores que han desarrollado funciones por un extenso periodo, permitiéndoles -previa ponderación de los valores del proyecto Nación- obtener la estabilidad en el empleo a diciembre de 2022, teniendo en cuenta que deben completar el plazo de 12 meses del artículo 17, inciso a, de la Ley 25.164

\section{Formación para la gestión pública}

Los procesos formativos públicos se inscriben en una disputa de sentidos sobre el rol y la orientación del Estado: la integración y la igualdad social, la centralidad del Estado, el enfoque de DDHH, género y diversidades sexuales. Debemos construir una Administración pública para todas y todos, donde trabajadores/as y decisores/as políticos/as puedan desarrollarse brindando servicios al pueblo y representando su voluntad soberana. 
Los procesos formativos requieren de la conducción estratégica y definiciones políticas de quienes representan la voluntad democráticamente expresada, más allá del trabajo conjunto que se desarrolle con Universidades públicas, sindicatos u organizaciones sociales.

Una debilidad que puede observarse en la formación estatal radica en la multiplicidad de áreas de formación-capacitación que no siempre coordinan sus acciones y estrategias. Por otra parte, en muchos casos esta formación se reduce a aspecto legales, técnicos u operativos de la gestión. Es clave articular/homogeneizar las estrategias formativas para garantizar la unidad de concepción, sin perjuicio de las especificidades de cada organismo. Las actividades de formación deben, además, pensarse con un enfoque federal que ponga en valor los recursos provinciales y municipales.

\section{Propuestas para un gobierno de todxs}

En materia de capacidades político estratégicas y desarrollo:

- Poner en valor los procesos de planificación estratégica y gestión en los territorios a través de la articulación del COFEPLAN con el INAP, en particular, y con la Jefatura de Gabinete de Ministros, en general. En este marco, crear ámbitos de Planificación Estratégica en cada organismo estatal.

- Diseñar un programa de formación para la comunicación efectiva, con el objetivo de garantizar la comunicación de las acciones estatales y una respuesta rápida a situaciones de desinformación o noticias falsas, mediante espacios abiertos para la resolución colectiva de problemas donde se fomenten los valores públicos y el compromiso con la transparencia, la información de calidad y el bienestar del pueblo.

- En este marco, conformar una unidad para la respuesta comunicacional efectiva, con la función de producir y suministrar información veraz, adecuada y suficiente sobre asuntos de interés social preestablecidos (salud, empleo, conciencia tributaria, educación, etc.) que sean objeto de desinformación o noticias falsas. Proponemos renovar las estrategias comunicacionales y discursivas para mejorar la gobernabilidad y la sostenibilidad de las medidas políticas. Se trata de planificar la "batalla cultural". 
- Establecer un Ente Planificador para el Desarrollo Territorial, orientado al logro de metas ambiciosas en el marco de la estrategia de gobierno y la mejora de la coordinación entre organismos que, en ocasiones, actúan de manera fragmentaría y poco articulada.

- Construir una agenda activa y coordinada con las Universidades Nacionales para potenciar su rol como instituciones articuladoras de las demandas locales para el desarrollo territorial.

- En este marco, implementar una Ventanilla de Información y Promoción Productiva, para acceder a la información sobre los distintos instrumentos de promoción existentes a nivel nacional, donde se brinde asistencia y asesoramiento, en particular, para las PyMEs.

En materia de gestión federal:

- Potenciar el rol, las capacidades y funciones del Consejo Federal de Inversiones (CFI), institución clave para las relaciones federales y el apoyo a las provincias.

- Consolidar y jerarquizar los Consejos Federales, ampliando sus funciones de acuerdo sectorial para el diseño y despliegue de políticas públicas con espíritu federal, incluyendo la aprobación de una ley para su organización y funcionamiento.

- Promover Acuerdos Regionales Metropolitanos, que constituyen un capítulo especial de la coordinación entre Nación, provincias y municipios. Es necesario generar instancias de coordinación de políticas territoriales para estas áreas.

- Atender a la cuestión de los grandes conglomerados poblacionales, la producción de suelo urbano, logística, transporte y otras, que la pandemia ha puesto en agenda, reactualizando los desequilibrios demográficos del país. Las estrategias para 
enfrentar la problemática deben partir de acuerdos metropolitanos como fruto del cruce virtuoso entre lo territorial y lo institucional.

- Fortalecer la formación federal a través de una Ley Nacional de Formación Pública que incluya la creación de una Red Federal de Escuelas de Gobierno y Administración Pública, en articulación con el sistema educativo formal y la red de universidades públicas. (Ver propuesta final Ley Scalabrini Ortiz)

En materia de capacidades institucionales y regulatorias:

- Avanzar y profundizar el camino de la agilización de trámites, tal como hoy sucede en trámites emblemáticos del RENAPER y ANSES, con un ámbito para el estudio de la normativa procedimental, para la detección de exigencias que perdieron sentido y pueden suplirse mediante nuevas tecnologías, y articulación interadministrativa. Implementar un programa de optimización de procesos y trámites públicos para asistir a las diversas jurisdicciones en su análisis, evaluación y diagnóstico, fortaleciendo sus capacidades de diseño de procesos y promoviendo la coordinación interadministrativa con una mirada sistémica.

- Desarrollar un programa de colaboración con la comunidad para mejorar la interacción de personas y organizaciones comunitarias, sociales y productivas con la administración, articulando: puntos de atención comunitaria centralizada, presenciales y virtuales; integración e interoperabilidad interna de estructuras y sistemas; $y$ procuradores comunitarios que serán el nexo con las unidades de responsabilidad primaria en la resolución y respuesta oportuna a la comunidad.

En materia de compras y contrataciones:

- Revisar la normativa procedimental y los trámites que han perdido vigencia, para su readecuación. 
- Integrar los procesos de compras y contrataciones a la gestión organizacional, a partir de una revisión integral de los sistemas que están operando.

- Readecuar la capacitación, con una mirada que integre las áreas de compras y contrataciones con las áreas de presupuesto y las áreas técnicas, acoplando los procesos contractuales a la planificación presupuestaria.

- Flexibilizar y diseñar mecanismos para el trabajo en red, promoviendo que cada organización tenga una política de desarrollo de proveedores.

- Promover un cupo de compras a empresas nacionales, facilitándoles los procesos y mejorando su interacción con las áreas de compras y los organismos que apoyan el desarrollo de la producción, aplicando los avances del sistema científico (investigación aplicada con concurrencia de INTI, INTA y otros). Reeditar, con vistas al desarrollo territorial equilibrado y la reactivación productiva, el concepto de compre nacional (programa Argentina compra).

En materia de empleo público:

- Revisar el procedimiento y de las normas sobre concursos (CCT 214/2006 y sectoriales, Resolución SGP 39/2010 y su modificatoria Resolución SEP 342/2019), a los efectos de realizar los procesos de selección y promoción evaluando en todas sus etapas capacidades ético-institucionales, técnico-profesionales y actitudinales. El perfil del trabajador/a estatal deberá considerar el apego por la democracia, la historia y realidad nacional y los derechos humanos, en la búsqueda permanente de un desarrollo con justicia social.

- Implementar procesos de búsqueda y selección para la contratación temporaria mediante publicidad y entrevistas ágiles que ponderen las tres tipos de capacidades. 
- Generar contenidos con el Consejo Federal de la Función Pública (CoFeFuP), articulando con el INAP, como manuales de gestión accesibles a cualquier ingresante al trabajo estatal.

- Revisar el enfoque de las evaluaciones laborales desde la perspectiva de las tres capacidades requeridas en el proceso de selección.

- Desarrollar un proceso de regularización especial, con formación ético institucional que permita conocer la historia y formación del Estado Argentino, su sistema federal y sus mandatos constitucionales en materia de derechos.

- Diseñar un programa específico de movilidad transitoria asociada a la estabilidad en el marco de salida de la pandemia.

- Evaluar el impacto producido en las y los trabajadores estatales por las políticas del Gobierno anterior y el aislamiento y el trabajo remoto durante el 2020, brindándoles herramientas necesarias para fortalecer sus roles y subjetividades de cara al 2021.

- Promover las trayectorias horizontales en las organizaciones públicas, logrando que el ejercicio o la pérdida de funciones directivas transitorias no sea un condicionante para el desarrollo de las capacidades y el trabajo digno de las y los trabajadores estatales en el marco de los proyectos institucionales.

- Abrir oportunidades a sectores más postergados, especialmente jóvenes, con opciones de terminalidad de estudios formales.

En materia de formación:

- Mapear los ámbitos de formación de las carteras y organismos públicos. Revisar los programas de formación que aún se fundan en la NGP 
o el reglamentarismo burocrático, rediseñando sus programas en diálogo con las organizaciones de trabajadores/as.

- Unificar, en materia de definición de objetivos y metas estratégicas, el diseño de programas de formación para la dirección y gestión pública.

- Ley Scalabrini Ortiz. Elaborar una ley de formación para la gestión pública estatal, en homenaje a Raúl Scalabrini Ortiz, donde se establezca como condición para asumir un cargo público y/o para el ingreso o la promoción laboral en el sector público, la realización de un curso de inducción que garantice niveles mínimos de conocimiento en: 1) historia nacional y latinoamericana. Conformación de los estados latinoamericanos. 2) estructura socioeconómica nacional; 3) pensamiento social situado; 4) elementos, dispositivos, herramientas y modalidades para una gestión pública inclusiva, nacional, popular y con perspectiva de DDHH y género, incluyendo la organización estatal, su rol, construcción histórica, perspectivas ideológicas y simbólico-culturales, y procesos técnico-administrativos básicos.

Comisión Estado y Administración Pública del Instituto Patria.

Diciembre de 2020. 
${ }^{1}$ Discurso pronunciado el 2 de julio de 1952 ante los altos funcionarios de la Administración Nacional.

${ }^{2}$ La coordinación general de la Comisión estuvo a cargo de la Dra. Claudia Bernazza, diputada nacional y docente de la Universidad Nacional de Lanús (nota de la RPPP).

${ }^{3}$ Discurso pronunciado por el Presidente Juan D. Perón, el 2 de julio de 1952, ante los altos funcionarios de la Administración Nacional. 